\title{
A note on the distribution of normalized prime gaps
}

\author{
János Pintz*
}

\section{Introduction}

The Prime Number Theorem implies that the average value of

$$
d_{n}=p_{n+1}-p_{n}
$$

is $(1+o(1)) \log p_{n}$ if $n \in[N, 2 N]$, for example, where $\mathbb{P}=\left\{p_{i}\right\}_{i=1}^{\infty}$ is the set of primes. This motivates the investigation of the sequence $\left\{d_{n} / \log p_{n}\right\}_{n=1}^{\infty}$ or $\left\{d_{n} / \log n\right\}_{n=1}^{\infty}$ (which is asymptotically equal). Erdős formulated the conjecture that the set of its limit points

$$
J=\left\{\frac{d_{n}}{\log n}\right\}^{\prime}=[0, \infty]
$$

He writes in [Erd 1955]: "It seems certain that $d_{n} / \log n$ is everywhere dense in $(0, \infty)$ " (after mentioning the conjecture $\liminf _{n \rightarrow \infty} d_{n} / \log n=0$ ). The fact that $\infty \in J$ was proved already in 1931 by Westzynthius [Wes 1931].

In 2005 Goldston, Yildırım and the author [GPY 2006], GPY 2009] showed $0 \in J$ which is the hitherto only concrete known element of $J$. On the other hand already 60 years ago Ricci Ric 1954 and Erdős [Erd 1955] proved (simultaneously and independently) that $J$ has a positive Lebesgue measure. A partial result towards the full conjecture (1.2) was shown by the author in [Pin 2013arX] according to which there exists an ineffective constant $c$ such that

$$
[0, c] \subset J .
$$

\footnotetext{
*Supported by OTKA Grants NK104183, K100291 and ERC-AdG. 321104.
} 
In a recent work W. Banks, T. Freiberg and J. Maynard BFM 2014arX] proved that for any sequence of $k=9$ nonnegative real numbers $\beta_{1} \leqslant \beta_{2} \leqslant$ $\cdots \leqslant \beta_{k}$ we have

$$
\left\{\beta_{j}-\beta_{i}: 1 \leqslant i<j \leqslant k\right\} \cap J \neq \emptyset .
$$

As a corollary they obtained that if $\lambda$ denotes the Lebesgue measure, then

$$
\lambda([0, T] \cap J) \geqslant(1+o(1)) T / 8 .
$$

\section{Generalization and Improvement}

The purpose of this note is to generalize this result for the case when $d_{n}$ is normalized by a rather general function $f(n) \rightarrow \infty$, that is to consider instead of $J$ the more general case of the set of limit points

$$
J_{f}=\left\{\frac{d_{n}}{f(n)}\right\}^{\prime}
$$

where we require from $f$ to belong to the class $\mathcal{F}$ below.

Definition. A function $f(n) \nearrow \infty$ belongs to $\mathcal{F}$ if for any $\varepsilon>0$

$$
(1-\varepsilon) f(N) \leqslant f(n) \leqslant(1+\varepsilon) f(N) \text { for } n \in[N, 2 N], N>N_{0},
$$

further if

$$
f(n) \ll \log n \log _{2} n \log _{4} n /\left(\log _{3} n\right)^{2}
$$

where $\log _{\nu} n$ denotes the $\nu$-times iterated logarithm.

The first condition means that $f(n)$ is slowly oscillating, while the second one that it does not grow more quickly than the Erdős-Rankin function, which until the recent dramatic new developments by Maynard [May 2014arX], Ford-Green-Konyagin-Tao [FGKT 2014arX], and Ford-Green-KonyaginMaynard-Tao [FGKMT 2014arX] described the largest known gap between consecutive primes. The improvement means that it is sufficient to work with $k=5$ values of $\beta_{i}$ in (1.4) instead of $k=9$ values. As an immediate corollary we obtain a lower bound $(1+o(1)) T / 4$ instead of (1.5) for the Lebesgue measure of the more general set $[0, T] \cap J_{f}$. 
Theorem 1. If $f \in \mathcal{F}$, then for any sequence of $k=5$ nonnegative real numbers $\beta_{1} \leqslant \beta_{2} \leqslant \cdots \leqslant \beta_{k}$ we have

$$
\left\{\beta_{j}-\beta_{i}: 1 \leqslant i<j \leqslant k\right\} \cap J_{f} \neq \emptyset .
$$

Corollary 2. If $f \in \mathcal{F}$, then

$$
\lambda([0, T] \cap J) \geqslant(1+o(1)) T / 4 .
$$

In an earlier work Pin 2013arX] we showed that for any $f \in \mathcal{F}$ there exists an ineffective constant $c_{f}$ such that $\left[0, c_{f}\right] \subset J_{f}$. We further remark that since $\beta_{i}$ can be arbitrarily large, Theorem 1 includes the improvement of the Erdös-Rankin function given in (2.3) proved recently in May 2014arX and [FGKT 2014arX]. (We note that the proof uses some refinement of the argument of May 2014arX], so it does not represent an independent new proof.)

In connection with the original Erdős conjecture for general $f \in \mathcal{F}$ we remark that it was proved in Pin 2014arX] that the conjecture is in some sense valid for almost all functions $f \in \mathcal{F}$. More precisely it was shown in [Pin 2014arX] that if $\left\{f_{n}\right\}_{1}^{\infty} \in \mathcal{F}$ with $\lim _{x \rightarrow \infty} f_{n+1}(x) / f_{n}(x)=\infty$, then

$$
J_{f_{n}}=[0, \infty]
$$

apart from at most 98 exceptional functions $f_{n}$.

\section{Proof}

The generalization for the case $f \in \mathcal{F}$ instead of the single case $f=\log n$ runs completely analogously to the proofs in [Pin 2014arX] so we will only describe how to improve $k=9$ to $k=5$ in Theorem 1 which leads to the improved Corollary 2 in the same simple way as described in the Introduction of the work of Banks, Freiberg and Maynard [BFM 2014arX].

The result will follow from the following improvement of Theorem 4.3 of their work. Let $\mathcal{Z}$ be given by (4.8) of [BFM 2014arX].

Theorem 3. Let $m, k$ and $\varepsilon=\varepsilon(k)$ be fixed. If $k$ is a sufficiently large multiple of $4 m+1$ and $\varepsilon$ is sufficiently small, there is some $N(m, k, \varepsilon)$ such that the following holds for $N \geqslant N(m, k, \varepsilon)$ with

$$
w=\varepsilon \log N, \quad W=\prod_{p \leqslant w, p \nmid \mathcal{Z}} p .
$$


Let $\mathcal{H}=\left\{h_{1}, \ldots, h_{k}\right\}$ be an admissible $k$-tuple (that is it does not cover all residue classes mod $p$ for any prime $p$ ) such that

$$
0 \leqslant h_{1}<\cdots<h_{k} \leqslant N
$$

and

$$
p \mid \prod_{1 \leqslant i<j \leqslant k}\left(h_{j}-h_{i}\right) \Longrightarrow p \leqslant w .
$$

Let $\mathcal{H}=\mathcal{H}_{1} \cup \cdots \cup \mathcal{H}_{4 n+1}$ be a partition of $\mathcal{H}$ into $4 m+1$ sets of equal size and let $b$ be an integer with

$$
\left(\prod_{i=1}^{k}\left(b+h_{i}\right), W\right)=1 .
$$

Then there is some $n \in(N, 2 N]$ with $n \equiv b(\bmod W)$ and some set of distinct indices $\left\{i_{1}, \ldots, i_{m+1}\right\} \subseteq\{1, \ldots, 4 m+1\}$ such that

$$
\left|\mathcal{H}_{i}(n) \cap \mathbb{P}\right| \geqslant 1 \quad \text { for all } i \in\left\{i_{1}, \ldots, i_{m+1}\right\} .
$$

Remark. The original analogous statement (4.20) of [BFM 2014arX] should have been stated with $\geqslant 1$ instead of $=1$ (oral communication of James Maynard). This form is enough to imply their Corollary 1.2 or our Corollary 2 .

The needed change in the Deduction of Theorem 4.3 is the following. First, using $4 m+1 \mid k$ we write

$$
\mathcal{H}=\mathcal{H}_{1} \cup \cdots \cup \mathcal{H}_{4 m+1}
$$

as a partition of $\mathcal{H}$ into $4 m+1$ sets each of size $k /(4 m+1)$. Instead of the quantity $S$ in [BFM 2014arX] we introduce with a new parameter $\alpha=\alpha(m)$ the new quantity $S(\alpha)$, where $\alpha$ will be chosen relatively small (we will see that $\alpha(m)=1 /(5 m)$ is a good choice, for example). Thus, let with a further parameter $\beta$

$$
\begin{aligned}
S(\alpha, \beta)= & \sum_{N<n \leqslant 2 N}\left(\sum_{i=1}^{k} 1_{\mathbb{P}}\left(n+h_{i}\right)-\beta m-\alpha \sum_{j=1}^{4 m+1} \sum_{\substack{h, h^{\prime} \in \mathcal{H}_{j} \\
h \neq h_{2}^{\prime}}} 1_{\mathbb{P}}(n+h) 1_{\mathbb{P}}(n+h)^{\prime}\right) \\
& \times\left(\sum_{\substack{d_{1}, \ldots, d_{k} \\
d_{i} \mid n+h_{i} \forall i}} \lambda_{d_{1}, \ldots, d_{k}}\right)^{2}
\end{aligned}
$$


where under the summation sign we consider unordered pairs $h, h^{\prime} \in \mathcal{H}_{j}$. Let

$$
\beta=\beta(\alpha)=\max _{\ell \in \mathbb{Z}^{+}}\left(\ell-\alpha\left(\begin{array}{l}
\ell \\
2
\end{array}\right)\right) .
$$

Then the contribution of any set $\mathcal{H}_{j}$ to $S(\alpha, \beta)=S(\alpha)$ is at most $\beta$, so if we have for every $n \in(N, 2 N]$ at most $m$ sets of the form $\mathcal{H}_{j}$ with

$$
\sum_{h \in \mathcal{H}_{j}} 1_{\mathbb{P}}(n+h)>0,
$$

then consequently

$$
S(\alpha) \leqslant 0 .
$$

In contrary to the choice $\varrho \in(0,1)$ and $\delta \varrho \log k=2 m$ of [BFM 2014arX] we will choose now $\delta \varrho \log k$ much larger

$$
\delta \varrho \log k=u:=\frac{4 m+1}{4 \alpha}, \quad \alpha=\frac{1}{5 m}, \quad \varrho \in(0,1) .
$$

This implies with an easy calculation

$$
\beta=\frac{5 m+1}{2}
$$

Using the same argument for the estimation of the negative double sum as [BFM 2014arX] we obtain a choice of a function $F$ such that

$$
\begin{aligned}
& S(\alpha)=\frac{N}{W} B^{-k} I_{k}(F)\left(\sum_{i=1}^{k} \frac{u}{k}(1+O(\gamma))-\beta m-4 \alpha \sum_{j=1}^{4 m+1} \sum_{\substack{h, h^{\prime} \in \mathcal{H}_{j} \\
h \neq h^{\prime}}} \frac{u^{2}}{k^{2}}(1+O(\delta+\gamma))\right) \\
& =\frac{N}{W} B^{-k} I_{k}(F)\left(u(1+O(\gamma))-\frac{(5 m+1) m}{2}-\frac{4(4 m+1)}{5 m}\left(\begin{array}{c}
k /(4 m+1) \\
2
\end{array}\right) \frac{u^{2}}{k^{2}}(1+O(\delta+\gamma))\right) .
\end{aligned}
$$

By the above choice of the parameters in (3.11) we have from (3.13) with $\gamma=(\log k)^{-1 / 2}$

$$
\begin{aligned}
\frac{S(\alpha) W B^{k}}{N I_{k}(F)} \geqslant & \frac{5 m(4 m+1)(1+O(\gamma))}{4}-\frac{(5 m+1) m}{2} \\
& -\frac{m(1+O(m(\delta+\gamma)))}{8}>0,
\end{aligned}
$$


which contradicts to (3.10).

In order to see the validity of the last inequality we can choose

$$
m<(\log k)^{1 / 4} \Longleftrightarrow \delta \asymp\left(\frac{m^{2}}{\log k}\right)
$$

which implies $m \gamma=o(1)$ and $m \delta=o(1)$. This contradiction proves our Theorem 1. Corollary 2 follows from it in the same way as Corollary 1.2 from Theorem 1.1 in [BFM 2014arX].

\section{References}

[BFM 2014arX] W. D. Banks, T. Freiberg, J. Maynard, On limit points of the sequence of normalized prime gaps, arXiv: $1404.5094 \mathrm{v} 2$ [math. NT] 20 Oct. 2014.

[Erd 1955] P. Erdős, Some problems on the distribution of prime numbers, Teoria dei Numeri, Math Congr. Varenna, 1954, 8 pp., 1955.

[FGKT 2014arX] K. Ford, B. Green, S. Konyagin, T. Tao, Large gaps between consecutive prime numbers, arXiv:1408.4505, 20 Aug. 2014.

[FGKMT 2014arX] K. Ford, B. Green, S. Konyagin, J. Maynard, T. Tao, Long gaps between primes, arXiv:1412.5029v1, 16 Dec. 2014.

[GPY 2009] D. A. Goldston, J. Pintz and C. Y. Yıldırım, Primes in tuples I, Ann. of Math. (2) $\mathbf{1 7 0}$ (2009), 819-862.

[GPY 2006] D. A. Goldston, J. Pintz, C. Yildirim, Primes in Tuples III: On the difference $p_{n+\nu}-p_{n}$. Funct. Approx. Comment. Math. 35 (2006), 79-89.

[May 2014arX] J. MAYNARD, Large gaps between primes, arXiv: 1408.5110 [math. NT], 21 Aug. 2014.

[Pin 2013arX] J. Pintz, Polignac Numbers, Conjectures of Erdős on Gaps between Primes, Arithmetic Progressions in Primes, and the bounded Gap Conjecture, arXiv: 1305.6289v1 [math. NT], 27 May 2013, to appear in: Memorial Volume for Wolfgang Schwarz, Springer. 
[Pin 2014arX] J. Pintz, On the distribution of gaps between consecutive primes, arXiv:1407.2213v1, 8 July 2014.

[Ric 1954] G. Ricci, Sull'andamento della differenza di numeri primi consecutivi, Riv. Mat. Univ. Parma 5 (1954), 3-54.

[Wes 1931] E. Westzynthius, Über die Verteilung der Zahlen, die zu der $n$ ersten Primzahlen teilerfremd sind, Comm. Phys. Math. Helsingfors (5) 25 (1931), 1-37.

János Pintz

Rényi Mathematical Institute

of the Hungarian Academy of Sciences

Budapest, Reáltanoda u. 13-15

H-1053 Hungary

e-mail: pintz.janos@renyi.mta.hu 